\title{
Beer Taste Detection Based on Electronic Tongue
}

\author{
Hongmei Zhang, ${ }^{*}$ Guangyu Zou, Wanru Liu, and Zheng Zhou \\ College of Mechanical and Electrical Engineering, Henan Agricultural University, Zhengzhou 450002, China
}

(Received November 15, 2019; accepted April 1, 2020)

Keywords: electronic tongue, beer, principal component analysis (PCA), linear discriminant analysis (LDA), BP neural network

We attempted to detect the five tastes in four different commercially available brands of beer using an electronic tongue and conducted a statistical analysis on their alcohol contents, original wort concentrations, and $\mathrm{pH}$ values. Statistical methods, including principal component analysis (PCA), linear discriminant analysis (LDA), and a backpropagation (BP) neural network, were used to identify and classify the four beer brands. According to PCA, in the five taste indicators of the four brands of beer, the contribution rates of the first and second principal components were 56.73 and $34.46 \%$ respectively; the beer was oxidized to a certain extent with increasing detection time. The results of LDA confirmed the high sensitivity of the electronic tongue sensors to beer tastes as the four brands were effectively identified by distinguishing the taste differences among them. The results of the BP neural network suggested that its predictive accuracy for the five tastes in the four brands can achieve $100 \%$ subject to the conformity between the measured and predicted values. The stepwise regression model established in our study could be effective for accurately predicting the original wort concentration of beer. The determination coefficients of the original wort concentration modeling set and the validation set were 0.99 and 0.96 , and the root-mean-square errors were 0.06 and 0.41 , respectively. As demonstrated by its high sensitivity in analyzing the tastes of four different beer brands, the electronic tongue can effectively distinguish the taste differences among different beers.

\section{Introduction}

Beer is a brewed beverage made from malt, hops, and water as the main raw materials via the fermentation of yeast. ${ }^{(1)}$ Being rich in various nutrients required by the human body, ${ }^{(2)}$ beer has a complicated taste. Beer quality is usually detected through sensory evaluation and physicochemical indexes. ${ }^{(3,4)}$ Despite its relatively wide application in beer detection, sensory evaluation appears to be susceptible to external interference and influence from subjective factors; by contrast, physicochemical detection may be limited by its high cost, long cycle, and relatively simple characterization indexes. ${ }^{(5,6)}$ To provide a scientific and objective method for beer detection, the taste properties of beer were analyzed using an electronic tongue in our study.

*Corresponding author: e-mail: hmzh86022625@sina.com

https://doi.org/10.18494/SAM.2020.2710 
An electronic tongue sensor can produce different electrical signals in response to different adsorbed molecules, thereby simulating the biological taste system, enabling sample detection and analysis. An electronic tongue is mainly employed for liquid detection and has been applied to olive oil, ${ }^{(7,8)}$ tea, ${ }^{(9-11)}$ coffee, ${ }^{(12,13)}$ and other products. Several studies have investigated the applications of an electronic tongue in beer detection. Nery and Kubota ${ }^{(14)}$ invented a paperbased electronic tongue that can distinguish different brands and types of beer. Blanco et al. ${ }^{(15)}$ applied a portable electronic tongue based on an electrochemical screen-printed electrode array to beer analysis, and the results show that the discriminant model they established can classify beer with $100 \%$ accuracy. Deng et al. ${ }^{(16)}$ classified 10 types of commercially available beer and showed that an electronic tongue can effectively distinguish these 10 types of beer via principal component analysis (PCA). Li et al. ${ }^{(17)}$ conducted taste detection on 15 types of beer from six manufacturers using an electronic tongue and found that there were significant differences among different brands, origins, and categories of beer. By detecting beer and mixed beer samples, Jia et al. ${ }^{(6)}$ found that a partial least-squares model established on the basis of the beer taste could accurately predict the beer-mixing proportion.

In our study, an SA402B electronic tongue was used to detect the tastes of four beer brands. MATLAB software was adopted for PCA, linear discriminant analysis (LDA), and backpropagation (BP) neural network analysis to classify and identify these brands. Furthermore, on the basis of the five basic tastes of sourness, bitterness, astringency, umami, and saltiness detected by the electronic tongue, a multivariate linear regression model was established to predict the original wort concentration of beer.

\section{Materials and Methods}

\subsection{Experimental materials}

Four commercially available beer brands were used in the experiment: Snow Beer (A), Harbin Beer (B), Tsingtao (C), and Budweiser (D). To minimize the influence of different brewing and storage times on beer taste, the production dates of these four brands were very close.

\subsection{Instruments and equipment}

The electronic tongue used in our experiment was the SA402B taste-sensing system manufactured by INSENT Corporation, Japan. The sensor array consisted of five taste sensors and two reference electrodes. Upon sample testing, the electronic tongue can acquire the changes in the membrane potential of the sensors then transfer the signal to a computer. The measured potential values are automatically converted into taste values via the self-equipped electronic tongue signal-processing software in the computer. In addition to distinguishing the five basic tastes of sourness, bitterness, astringency, umami, and saltiness, the electronic tongue can also analyze bitterness, astringency, and umami in terms of their intensity. The sourness sensor can identify acidic substances in samples, such as citric acid and acetic acid, 
while the bitterness sensor usually responds strongly to acidic bitter substances. The astringent sensor is capable of detecting some astringent substances; the umami sensor can identify amino acids, nucleic acids, and other substances in samples; and the saltiness sensor can detect salts in samples such as sodium chloride and potassium chloride. Information on the sensors is given in Table 1.

\subsection{Experimental methods}

A total of eight samples (two replicate samples for each brand) were used for data collection with the electronic tongue. The sensors were washed in positive and negative solutions for $90 \mathrm{~s}$ and in two different standard solutions for $120 \mathrm{~s}$, which was followed by $30 \mathrm{~s}$ of self-testing. After testing each sample for $30 \mathrm{~s}$, the sensors were washed rapidly in two different standard solutions for $3 \mathrm{~s}$ before detecting the aftertaste. Eleven repetitions of the testing process were carried out for each sample.

\section{Results and Analysis}

\subsection{Analysis of beer basic information}

The basic information of the four beer brands is shown in Table 2. According to the table, the highest original wort concentration and alcohol content were found for Tsingtao Beer, followed by Budweiser, Harbin Beer, and Snow Beer. Furthermore, the original wort concentration was positively correlated with the alcohol content.

\subsection{Analysis of beer $\mathrm{pH}$ value}

The $\mathrm{pH}$ values of the sample solutions from the four beer brands were detected using a pentype $\mathrm{pH}$ meter. The detection was repeated three times for each brand and the means were calculated. The results are shown in Table 3. The table demonstrated no clear differences

Table 1

Taste sensor information of electronic tongue.

\begin{tabular}{ll}
\hline Sensor name & Sensor response characteristic \\
\hline Sourness sensor (CA0) & Sensitive to sourness \\
Bitterness sensor (C00) & Sensitive to bitterness \\
Astringency sensor (AE1) & Sensitive to astringency \\
Umami sensor (AAE) & Sensitive to umami \\
Saltiness sensor (CT0) & Sensitive to saltiness \\
\hline
\end{tabular}

Table 3

$\mathrm{pH}$ values of beer samples.

\begin{tabular}{lc}
\hline Sample No. & pH value \\
\hline A & 4.2 \\
B & 4.1 \\
C & 4.3 \\
D & 4.3 \\
\hline
\end{tabular}

Table 2

Beer sample information.

\begin{tabular}{lcc}
\hline Sample No. & $\begin{array}{c}\text { Original wort } \\
\text { concentration }\left({ }^{\circ} \mathrm{P}\right)\end{array}$ & $\begin{array}{c}\text { Alcohol content } \\
(\% \text { vol })\end{array}$ \\
\hline A & 8 & $\geq 2.5$ \\
B & 9 & $\geq 3.3$ \\
C & 10 & $\geq 4.0$ \\
D & 9.7 & $\geq 3.6$ \\
\hline
\end{tabular}


between the four brands; their $\mathrm{pH}$ values all fell in the range of 4.1-4.3, suggesting that the $\mathrm{pH}$ value of beer is not significantly correlated with the original wort concentration or alcohol content. The factors affecting $\mathrm{pH}$ are usually associated with beer ingredients and the brewing environment. Meanwhile, the $\mathrm{CO}_{2}$ contained in the water and the yeast activity may also have a certain impact. The sour taste of beer may be affected by its $\mathrm{pH}$ to some extent. In our case, however, the $\mathrm{pH}$ values of the four brands were relatively close and thereby failed to serve as a standard for distinguishing beers.

\subsection{Analysis of beer taste using electronic tongue}

The differences among the four beer brands as detected by the electronic tongue taste sensors in terms of the five basic tastes and three aftertastes are shown in Fig. 1. The taste differences among the four beer brands might be related to their different production processes, raw materials, varieties, and sources. The refreshing taste and lingering aftertaste of beer are mainly based on the sour and bitter tastes. The sourness of beer is affected by factors such as the temperature and environmental carbon dioxide content in the area of production during brewing, whereas the bitterness may be associated with bitter peptides in the brewing process as well as ingredients such as picric acid from hops. The chemical components accounting for the bitter taste include tannins and other compounds. ${ }^{(18)}$ Moreover, the diverse brewing customs in different regions, which can lead to differences in the loading procedure of raw materials, will also result in differences in bitterness.

The correlation between different taste indexes of the beer samples obtained by our analysis is shown in Table 4. According to the data, there was some correlation between the different tastes in these beer samples. Among them, sourness was found to have a significant negative

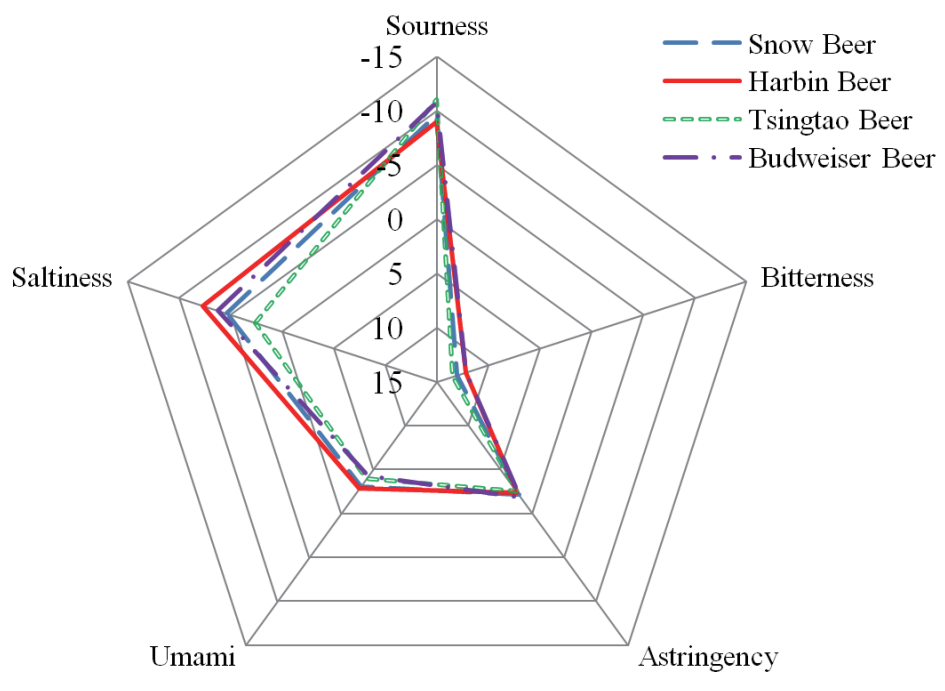

Fig. 1. (Color online) Radar diagram of electronic tongue sensor response. 
Table 4

Correlation analysis of beer tastes.

\begin{tabular}{lccccc}
\hline Taste & Sourness & Bitterness & Astringency & Umami & Saltiness \\
\hline Sourness & 1 & & & & \\
Bitterness & $-0.410^{* *}$ & 1 & & & \\
Astringency & 0.121 & $0.513^{* *}$ & 1 & & \\
Umami & $-0.964^{* *}$ & 0.168 & $-0.285^{*}$ & 1 & \\
Saltiness & $-0.660^{* *}$ & $0.894^{* *}$ & $0.381^{* *}$ & $0.465^{* *}$ & 1 \\
** represents high statistical significance $(p<0.01),{ }^{*}$ represents statistical significance $(p<0.05)$.
\end{tabular}

correlation with bitterness, umami, and saltiness. By contrast, a significant positive correlation was observed in the pairs of bitterness and astringency, bitterness and saltiness, and saltiness and umami. Furthermore, astringency was significantly negatively correlated with umami.

\subsection{PCA and LDA}

As a common processing method for data dimensionality reduction, PCA can convert a relatively large set of correlated indexes into a smaller set of uncorrelated comprehensive indexes called principal components via dimensionality reduction. Considering the high dimensionality of the data detected by the electronic tongue from the five tastes of the four beer brands, overlapping of the data was inevitable. PCA was able to reduce the overlapping highdimensional data and determine the weight of each coefficient.

The PCA results for the five electronic tongue sensors are shown in Fig. 2. In the PCA diagram, the greater the contribution rates of the horizontal and vertical coordinates PCA1 and PCA2, the better the principal components reflect the information of the original multiple indexes. ${ }^{(19)}$ According to Fig. 2, the contribution rate of PCA1 was $56.73 \%$ and that of PCA2 was $34.46 \%$, and their cumulative contribution rate was $91.19 \%$, indicating that PCA1 and PCA2 reflected the main information of the beer tastes. After dimensionality reduction, the aggregation effect of the four beer brands appeared to be distinct, suggesting that the electronic tongue sensors were able to effectively distinguish the four different brands of beer samples. The large intergroup gaps for Budweiser vs Tsingtao and Harbin Beer vs Tsingtao demonstrated major taste differences between Tsingtao and Budweiser and Harbin Beer. Correspondingly, the small intergroup gaps for Harbin Beer vs Snow Beer and Harbin Beer vs Budweiser implied minor taste differences. In the PCA diagram, the reduced gradient for the beer samples of the different brands might result from oxidization of the electronic tongue due to long-time exposure to the air, which thereby led to taste changes.

As a classification algorithm, the basic idea of LDA is to map samples to the optimal discriminant vector space so as to achieve the effect of reducing the feature space dimension and extracting classification information, thereby achieving the largest interclass gap and the smallest intraclass gap and thus give the model the best separability in this new subspace. ${ }^{(20)}$ LDA results of the taste data detected by the electronic tongue system are shown in Fig. 3. 


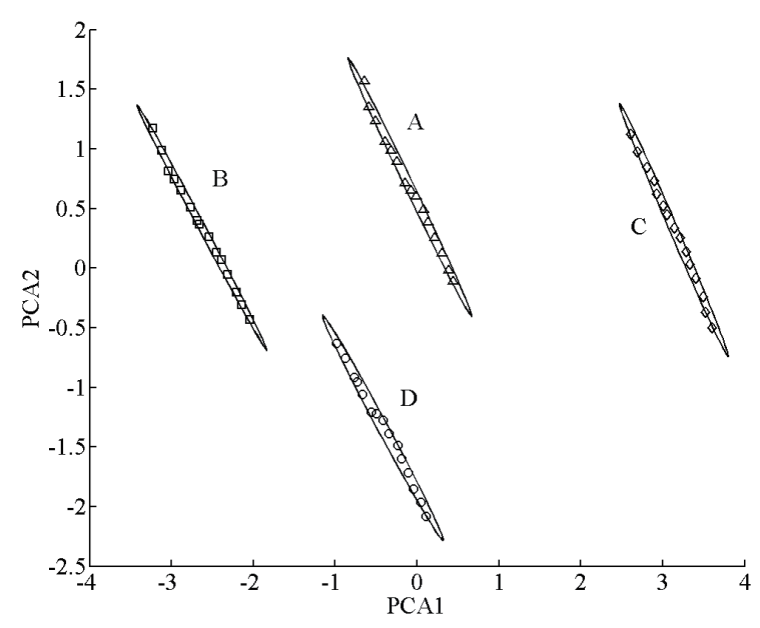

Fig. 2. PCA results of four brands of beer.

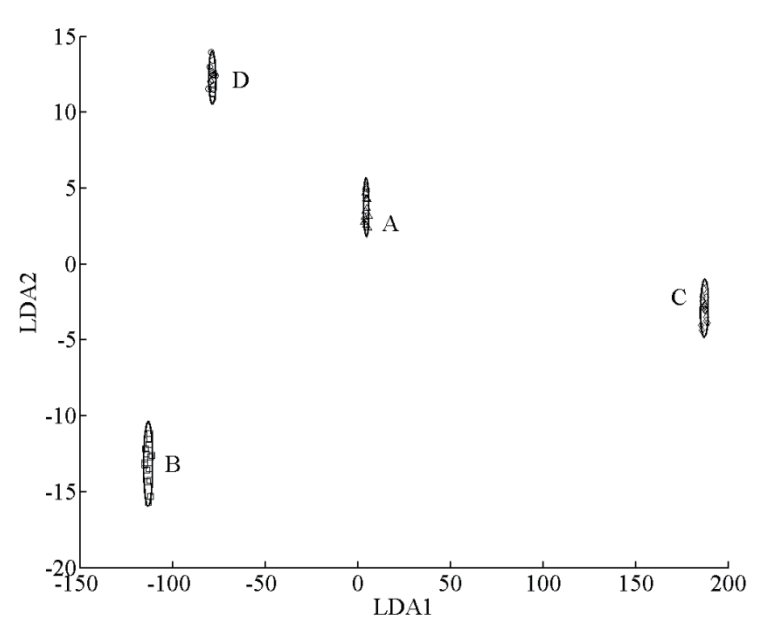

Fig. 3. LDA results of four brands of beer.

According to the results, the four beer brands can be clearly distinguished. Furthermore, the test sample points for each brand were compactly arranged, suggesting that LDA showed excellent ability to distinguish these four beer brands.

For the above data of the four beer brands obtained by the electronic tongue sensors, PCA was carried out to find an optimal mapping approach through analyzing and solving problems from the perspective of feature covariance, whereas LDA was performed to maximize the gap between different classes and increase the compactness of the arrangement of intraclass data points after mapping. ${ }^{(21)}$ Although both techniques exhibited good performance in distinguishing the four beer brands, LDA appeared to be more effective.

\subsection{BP neural network classification}

As a feedforward artificial neural network, a BP neural network consists of an input layer, a hidden layer, and an output layer and has the advantages of nonlinear mapping ability, generalization ability, and fault tolerance. ${ }^{(22-25)}$ A BP neural network has strong mapping ability of input values to output values while requiring no specific mathematical expressions to describe the mapping relationship between them. ${ }^{(26)}$ In our classification of the beers with the BP neural network, 10 sets of taste values were randomly selected from the beer samples as the training set, and the remaining five sets were taken as the verification set. With the 40 data items in the training set as the input layer of the BP neural network and the sample brands set as the output layer, the classification results are shown in Table 5. According to the sample detection output values, the trained neural network could correctly identify the different brands of beer. The BP neural network even successfully distinguished the brands with similar taste, with the recognition rates of training samples and verification samples both reaching $100 \%$, suggesting that the neural network met the required accuracy of identification for these four different brands. 
Table 5

BP neural network classification results of four brands of beer.

\begin{tabular}{lcc}
\hline Sample No. & $\begin{array}{c}\text { Recognition rate in } \\
\text { training samples (\%) }\end{array}$ & $\begin{array}{c}\text { Recognition rate in } \\
\text { verification samples (\%) }\end{array}$ \\
\hline A & 100 & 100 \\
B & 100 & 100 \\
C & 100 & 100 \\
D & 100 & 100 \\
Total & 100 & 100 \\
\hline
\end{tabular}

\subsection{Prediction of beer original wort concentration based on electronic tongue}

The composition of beer wort is complicated and mainly includes solids, sugar, nitrogenous substances, hop components, esters, tannins, sulfides, dissolved oxygen, phosphoric acid, and other substances. ${ }^{(27,28)}$ It has been shown that the original wort concentration in beer is an important factor affecting the taste; a lower original wort concentration will generate a thinner taste. ${ }^{(29,30)}$ In our study, the five tastes measured by the electronic tongue were used to predict the original wort concentration in beer, by which a multivariate linear regression model was established for the original wort concentration. In the modeling of the original wort concentration, sourness, bitterness, astringency, umami, and saltiness were taken as independent variables with the original wort concentration as the dependent variable. The regression model is given by Eq. (1), and the determination coefficients and root-mean-square (RMS) errors of the model are shown in Table 6. In this multivariate linear regression model, the regression coefficients were found to be significant, and the determination coefficients in the modeling set and verification set were both greater than 0.95 , confirming the excellent fitting effect. Moreover, the low RMS values of the modeling set and the verification set indicated little deviation between the predicted values and the actual values.

$$
M Z_{1}=27.334-0.38 x_{1}-1.845 x_{2}+2.134 x_{3}+0.764 x_{4}+0.417 x_{5}
$$

$M Z_{1}$ represents the original wort concentration; $x_{1}-x_{5}$ represent the taste values of sourness, bitterness, astringency, umami, and saltiness, respectively.

Possessing diverse tastes, the beer exhibited certain interactions between the five taste values of sourness, bitterness, astringency, umami, and saltiness. Table 4 also demonstrated significant correlations between some tastes. Therefore, in response to the interactions between tastes, interaction quadratic terms were added to the basic taste values to establish a stepwise regression model. In the modeling analysis for the original wort concentration, the five taste values and their interaction quadratic terms were taken as independent variables with the original wort concentration in the beer as the dependent variable. The regression model is given by Eq. (2), and the determination coefficients and RMS errors (RMSEs) of the model are shown in Table 7. In the model, the determination coefficients of the modeling set and validation set 
Table 6

Parameters of multivariate linear regression model.

\begin{tabular}{lcccc}
\hline \multirow{2}{*}{ Regression model } & \multicolumn{2}{c}{ Modeling set } & \multicolumn{2}{c}{ Verification set } \\
\cline { 2 - 5 } & $\begin{array}{l}\text { Determination } \\
\text { coefficient }\left(R^{2}\right)\end{array}$ & RMSE & $\begin{array}{l}\text { Determination } \\
\text { coefficient }\left(R^{2}\right)\end{array}$ & RMSE \\
\hline Multiple linear regression & 0.99 & 0.08 & 0.96 & 0.39 \\
\hline
\end{tabular}

Table 7

Parameters of stepwise regression model.

\begin{tabular}{lcccc}
\hline \multirow{2}{*}{ Regression model } & \multicolumn{2}{c}{ Modeling set } & \multicolumn{2}{c}{ Verification set } \\
\cline { 2 - 5 } & $\begin{array}{c}\text { Determination } \\
\text { coefficient }\left(R^{2}\right)\end{array}$ & RMSE & $\begin{array}{c}\text { Determination } \\
\text { coefficient }\left(R^{2}\right)\end{array}$ & RMSE \\
\hline Stepwise regression & 0.99 & 0.06 & 0.96 & 0.41 \\
\hline
\end{tabular}

were 0.99 and 0.96 , respectively, suggesting that its fitting effect was superior to that of the multiple linear regression model. The RMSEs in this model also turned to be lower, suggesting a smaller deviation between the predicted and measured values. Therefore, by comparing the two prediction models for the original wort concentration, the stepwise regression model was found to be superior.

$$
M Z_{2}=22.819-1.327 x_{2}+3.093 x_{3}+0.039 x_{1} x_{2}-0.099 x_{1} x_{4}+0.174 x_{3} x_{5}
$$

$M Z_{2}$ represents the original wort concentration; $x_{1}-x_{5}$ represent the taste values of sourness, bitterness, astringency, umami, and saltiness, respectively.

\section{Conclusions}

In this paper, the samples of four commercially available beer brands were subjected to index detection and analysis, and the results obtained are as follows.

There were significant differences in tastes among the different beer brands. The detection of correlation among the five tastes of beer demonstrated that sourness was significantly correlated with bitterness.

The different beer brands could be well distinguished according to the results of PCA and LDA dimensionality reduction of the taste data. The BP neural network exhibited excellent classification ability for the different beer brands, where the recognition rates of the training and validation sets reached $100 \%$.

According to the modeling analysis of the original wort concentration based on the five tastes of sourness, bitterness, astringency, umami, and saltiness, the original wort concentration had some influence on the beer taste. The multivariate linear regression and stepwise regression models established in our study were able to predict the original wort concentration. The better modeling effect was observed for the stepwise regression model, where the determination coefficients of the modeling set and the verification set were 0.99 and 0.96 , and the RMS errors were 0.06 and 0.41 , respectively. 
The electronic tongue has high sensitivity. Its sensitive "taste buds" can be used for the sensory evaluation of beer, which provides a new approach for expanding the beer market through developing beer with new tastes.

\section{Acknowledgments}

This work was financially supported by the Chinese National Foundation of Nature and Science through Project 31501213, the Special Fund for Henan Agriculture Research System through Project S2019-02-G07, Henan University Key Research Projects 20A210029, and the National Agricultural Industry Technology System through Project CARS-03.

\section{References}

1 C. A. Blanco, C. Andrés, and O. Monero: Crit. Rev. Food Sci. Nutr. 56 (2014) 1379. https://doi.org/10.1080/104 08398.2012.733979

2 J. J. Liu, J. L. Yang, X. Y. Zhang, B. Sun, X. T. Zhang, and H. Men: Trans. Chin. Soc. Agric. Mach. 47 (2016) 244. https://doi.org/10.6041/j.issn.1000-1298.2016.10.030

3 L. F. Castro and C. F. Ross: J. Inst. Brew. 121 (2015) 197. https://doi.org/10.1002/jib.219

4 M. Ghasemi, S. S. Mohtasebi, M. L. Rodriguez, J. Lozano, S. H. Razavi, H. Ahmadi, and C. Apetrei: Expert Syst. Appl. 39 (2012) 4315. https://doi.org/10.1016/j.eswa.2011.09.101

5 Y. Wang, J. G. Xing, J. Fu, and S. Qian: Transducer Microsyst. Technol. 37 (2018) 158. https://doi.org/10.13873/ j.1000-9787(2018)11-0158-03

6 H. F. Jia, A. H. Liang, J. H. He, L. J. Zhou, M. Zhang, and J. Z. Zheng: Food Sci. 32 (2011) 252. https://doi. org/10.1088/1674-4527/11/3/009

7 S. Slim, N. Rodrigues, and L. G. Dias: Eur. Food Res. Technol. 243 (2017) 1. https://doi.org/10.1007/s00217017-2856-8

8 T. H. P. Borges, A. M. Peres, L. G. Dias, and L. Seiquer: LWT Food Sci. Technol. 93 (2018) 150. https://doi. org/10.1016/j.lwt.2018.03.025

9 D. Chen, T. Ma, W. L. San, C. Wang, and Q. H. Li: Food Sci. 38 (2017) 168. https://doi.org/10.7506/spkx10026630-201718027

10 P. Saha, S. Ghorai, B. Tudu, and R. Bandyopadhyay: IEEE Sens. J. 16 (2016) 4470. https://doi.org/10.1109/ JSEN.2016.2544979

11 A. Ghosh, A. K. Bag, P. Sharma, and B. Tudu: IEEE Sens. J. 15 (2015) 6255. https://doi.org/10.1109/ JSEN.2015.2455535

12 T. C. B. D. Morais, D. Rodrigues, U. T. D. C. P. Souto, and S. G. Lemos: Food Chem. 273 (2018) 31. https://doi. org/10.1016/j.foodchem.2018.04.136

13 E. Várvölgyi, A. Gere, D. Szöllősi, and L. Sipos: Arabian J. Sci. Eng. 40 (2015) 125. https://doi.org/10.1007/ s13369-014-1489-5

14 E. W. Nery and L. T. Kubota: Anal. Chim. Acta 918 (2016) 60. https://doi.org/10.1016/j.aca.2016.03.004

15 C. A. Blanco, R. D. L. Fuente, I. Caballero, and M. L. Rodríguez: J. Food Eng. 157 (2015) 57. https://doi. org/10.1016/j.jfoodeng.2015.02.018

16 L. Deng, C. D. Pan, J. R. He, J. Shu, S. S. Xiao, and X. Chen: Anal. Instrum. 2 (2013) 63.

17 J. M. Li, Z. H. Zhang, Y. Zheng, Y. J. Guo, X. Li, and Q. Sun: Mod. Food 12 (2017) 115. https://doi. org/10.16736/j.cnki.cn41-1434/ts.2017.12.036

18 G. A. F. Harrison: J. Inst. Brew. 76 (2013) 486. https://doi.org/10.1002/j.2050-0416.1970.tb03333.x

19 Z. Haddi, A. Amari, H. Alami, and N. E. Bari: Sens. Actuators, B 155 (2011) 456. https://doi.org/10.1016/ j.snb.2010.12.047

20 M. Tohidi, M. Ghasemi, V. Ghafarinia, and S. S. Mohtasebi: Measurement 124 (2018) 120. https://doi. org/10.1016/j.measurement.2018.04.006

21 F. Y. Ma: Sci. Technol. Vision 13 (2015) 52. https://doi.org/10.19694/j.cnki.issn2095-2457.2015.13.032

22 S. Xu, E. L. Lü, H. Z. Lu, and Z. Y. Zhou: Sensors 16 (2016) 852. https://doi.org/10.3390/s16060852

23 Y. W. Wang, J. Wang, B. Zhou, and Q. J. Lu: Anal. Chim. Acta 650 (2009) 183. https://doi.org/10.1016/ j.aca.2009.07.049 
24 X. J. Peng and X. X. Weng: J Guangxi Norm. Univ. (Nat. Sci. Ed.) 35 (2017) 28 (in Chinese). https://doi. org/10.16088/j.issn.1001-6600.2017.01.005

25 C. Zhang, H. Ye, F. Liu, Y. He, W. W. Kong, and K. C. Sheng: Sensors 16 (2016) 244. https://doi.org/10.3390/ s16020244

26 J. Yao and J. Xu: Appl. Mech. Mater. 29 (2010) 2804. https://doi.org/10.4028/www.scientific.net/AMM.2932.2804

27 L. Hong, F. Gabriella, S. Kun, J. W. Wang, S. J. Yan, W. Y. Jin, and J. J. Chu: Liquor-Making Sci. Technol. 6 (2015) 16. https://doi.org/10.13746/j.njkj.2005.01.017

28 E. Bravi, P. Benedetti, O. Marconi, and G. Perretti: Food Chem. 215 (2017) 341. https://doi.org/10.1016/ j.foodchem.2016.07.153

29 S. Wu, K. L. Zhang, F. Sun, Y. D. Ju, and J. H. Du: Liquor Making 4 (2005) 44. https://kns.cnki.net/KCMS/ detail/detail.aspx dbcode $=$ CJFQ \&dbname $=$ CJFD2005\&filename $=$ NJZZ200504021\&v=MTE0NzdZUjhlWDF MdXhZUzdEaDFUM3FUcldNMUZyQ1VSN3FmWmVadEZpRGdVYjNCS31mUmRMRzRIdFRNcTQ5SFo=

30 M. Yano, W. Back, and M. Krottenthaler: J. Inst. Brew. 114 (2008) 357. https://doi.org/10.1002/j.2050-0416. 2008.tb00780.x

\section{About the Authors}

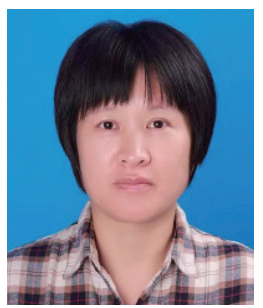

Hongmei Zhang received her B.S. and M.S. degrees from Henan Agricultural University, China, in 1999 and 2004, respectively, and her Ph.D. degree from Zhejiang University, China, in 2007. From 2007 to 2010, she was a lecturer at Henan Agricultural University. Since 2010, she has been an assistant professor at Henan Agricultural University. Her research interests are in nondestructive testing technology. (hmzh86022625@sina.com)

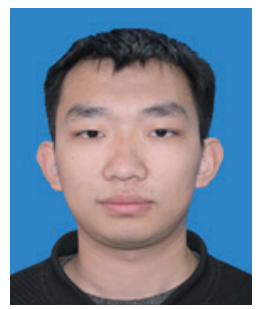

Guangyu Zou received his B.E. degree from Henan Agricultural University, China, in 2016. He is currently studying for his master's degree at Henan Agricultural University. His research interests are in the intelligent detection of agricultural products. (365786261@qq.com)

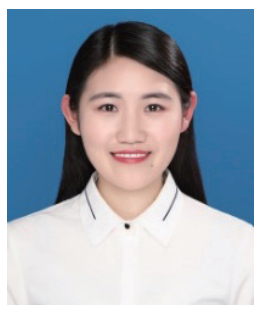

Wanru Liu received her B.E. degree from Henan Agricultural University, China, in 2018. Since 2018, she has been studying for a master's degree at Henan Agricultural University. Her research interests are in grain combine harvester design theory and methods. (18595850940@163.com)

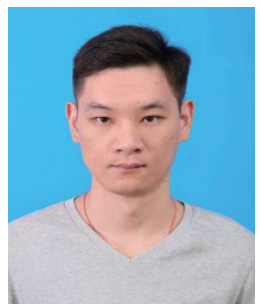

Zheng Zhou received his B.E. degree from Huanghe Science and Technology College, China, in 2019. Since 2019, he has been studying for a master's degree at Henan Agricultural University. His research interests are in corn threshing devices. (zhou609669165@qq.com) 